\section{PWE-011 INDICATIONS AND OUTCOMES OF PATIENTS RECEIVING IN-PATIENT PARENTERAL NUTRITION: TYPE 2 IF PATIENTS ON HPN}

Lauren O'Flynn*, Nicky Wyer, Nicola Burch. University Hospitals Coventry and Warwickshire NHS Trust, Coventry, UK

\subsection{6/gutjnl-2019-BSGAbstracts.342}

Introduction Parenteral nutrition (PN) enables nutritional requirements to be met in cases of intestinal failure (IF) where enteral nutrition is insufficient or not possible. ${ }^{1}$ The main objective of our audit was to evaluate the indication, outcome, and survival of patients who received PN during their acute hospital admission.

Methods Data on in-patients receiving PN was collected prospectively using the Nutrition Team database. This was then retrospectively analysed, including review of overall outcome and mortality within 12-months of the in-patient spell. All patients receiving PN as an in-patient between January 2014 and December 2017 were included.

Patients were categorised according to type of IF: T1IF (inpatient $\mathrm{PN}<28$ days); T2IF (in-patient $\mathrm{PN}>28$ days; or inpatient $\mathrm{PN}<28$ days who were discharged on HPN with a plan to electively restore intestinal continuity later); and T3IF admissions (established HPN patients admitted acutely). Here we discuss the results of patients with T2IF requiring acute in-patient admission.

Results A total of 117 T2IF admissions were identified, in 98 patients; 44\% male; age 1-3 years (mean 55). In 25 admissions the patient received $\mathrm{PN}$ for $<28$ days; however all were discharged as new HPN patients (16 T2IF; 9 T3IF (6 palliative)). Duration of $\mathrm{PN}$ in the remaining patients was 2-53 days (mean 52). Cumulative duration of all T2IF admissions was 5219 days. Assuming average cost of an NHS bed day of $£ 222$ (NICE 20152); this equates to $£ 1,158,618$ (approx. $£ 289,655 / \mathrm{yr})$ in bed days without considering cost of inpatient treatment. 12 patients had multiple admissions with T2IF (-, mean 2); $58 \%$ of these occurred in the same year, $42 \%$ in separate years.

Indication for PN included: fistulae (21\%); obstruction (13\%); short bowel (9\%); failure of enteral nutrition $(8 \%)$; post-surgical complications (7\%); dysmotility (7\%); ischaemic bowel (5\%); malabsorption (5\%); pancreatitis (4\%); cancer (4\%); anastamotic leak (3\%); perforation (3\%); post-op ileus (3\%); gastric outlet obstruction (3\%); no access for enteral nutrition (3\%); crohn's (1\%); pre-op nutrition (1\%); planned IF surgery (1\%).

Outcome of T2IF was discharge on HPN in 55\%. Outcome in the remaining patients included: oral nutrition $(26 \%)$; NJ/Jejunostomy (9\%); NG (6\%); and RIP on PN (4\%). 12month follow-up data was available in 109 patients; 12-month survival was 77\%; overall survival to end August 2018 was $71 \%(\mathrm{n}=83)$.

Conclusions This audit demonstrates the significant financial cost and bed burden to centres managing patients with T2IF; and highlights the need for an IF tariff. In $45 \%$ patients there was return of intestinal function and resolution of intestinal failure, highlighting reversibility of T2IF. It was perhaps surprising that 12-month survival in this cohort was lower than the sub-analysis of all T3IF in-patient admissions $(77 \%$ versus $62 \%)$; this likely reflects that acute in-patient admission in patients with T3IF is a sign of disease progression/decompensation and therefore an indicator for reduced 12-month survival.

\section{REFERENCES}

1. Loris Pironi, et al.Espen guidelines on chronic intestinal failure in adult. Clin Nutr 2016;35:24-07;

2.. Costing statement: Implementing the NICE guideline on Transition between inpatient hospital settings and community or care home settings for adults with social care needs (NG27)

\section{PWE-012 INDICATIONS AND OUTCOMES OF PATIENTS RECEIVING IN-PATIENT PARENTERAL NUTRITION: TYPE 3 IF PATIENTS ON HPN}

Lauren O'Flynn*, Nicky Wyer, Nicola Burch. University Hospitals Coventry and Warwickshire NHS Trust, Coventry, UK

\subsection{6/gutjnl-2019-BSGAbstracts.343}

Introduction Parenteral nutrition (PN) enables nutritional requirements to be met in cases of intestinal failure (IF) where enteral nutrition is insufficient or not possible. ${ }^{1}$ The main objective of our audit was to evaluate the indication, outcome, and survival of patients who received PN during their acute hospital admission.

Methods Data on in-patients receiving PN was collected prospectively using the Nutrition Team database. This was then retrospectively analysed, including review of overall outcome and mortality within 12-months of the in-patient spell. All patients receiving $\mathrm{PN}$ as an in-patient between January 2014 and December 2017 were included.

Patients were categorised according to type of IF: T1IF (inpatient $\mathrm{PN}<28$ days); T2IF (in-patient PN>28 days; or inpatient $\mathrm{PN}<28$ days who were discharged on HPN with a plan to electively restore intestinal continuity later); and T3IF admissions (established HPN patients admitted acutely). Here we discuss the results of patients with T3IF requiring acute in-patient admission.

Results A total of 55 in-patient admissions were identified in 26 existing HPN patients (16 female; 10 male). Age ranged from 2-0 years (mean 60). Cause of IF (indication for HPN) was: short bowel syndrome (40\%); dysmotility (38\%); palliative cancer (13\%); mesenteric ischaemia (7\%); and malabsorption $(2 \%)$.

Duration of in-patient episode was -2 days (mean 16; cumulative total 875). Assuming average cost of an NHS bed day of $£ 222$ (NICE 20152); this equates to $£ 194,250$ in bed days without considering cost of in-patient treatment. Number of admissions per patient ranged from - (mean 2).

Indication for admission included sepsis (35\%); disease flare (22\%); elective surgery (13\%); elective admission to commence HPN (9\%); electrolyte derangement (7\%); cancer progression (4\%); GI Bleed (4\%); chemotherapy complications $(2 \%)$; fractured pelvis (2\%); overdose (2\%); and tube change $(1 \%)$. Source of sepsis included: urinary $(n=6)$, chest $(n=5)$, CRBSI $(n=3)$, discitis $(n=2)$, cholecystitis $(n=2)$, and abdominal collection $(n=1)$. Elective surgery included: venting PEG $(n=1)$; GI surgery e.g. intestinal continuity $(n=4)$; and non-GI surgery $(n=2)$.

Outcome of admission in the majority was discharge on HPN ( $n=49 ; 89 \%)$; one stopped HPN following continuity surgery. A total of 4 patients died during the admission (7\%), and 1 was commenced on the 'care of the dying' pathway (2\%). 12-month follow-up data was available in 54 of the admissions (98\%); 1 patient had only 9-months following last admission at the time of analysis. 12-month survival was $62 \%$ 